\title{
Воздействие диэлектрического барьерного разряда на диатомит, загрязненный нефтепродуктами
}

\author{
(C) 2021 Гусев Г.И., Гущин А.А., Гриневич В.И., \\ Извекова Т.В., Шаронов А.В. \\ Ивановский государственный химико-технологический университет, Иваново
}

Поступила в редакцию 9.10.2020 г.

DOI: $10.17308 /$ sorpchrom.2021.21/3220

В последнее время внимание многих исследователей привлекают методы химии высоких энергий, в частности диэлектрический барьерный разряд (ДБР). Плазменно-адсорбционная очистка воды с последующей (или одновременной) регенерацией сорбентов с помощью ДБР является новой и малоизученной областью, поэтому исследование процессов обработки сорбентов в барьерном разряде является актуальной и важной задачей. Целью работы являлось выявление закономерностей процессов регенерации диатомита в плазме диэлектрического барьерного разряда, и особенностей воздействия плазмы на поверхностные свойства сорбента (диатомита марки СМД-Сорб) в реакторах ДБР планарного и коаксиального типов.

В работе использовались два реактора ДБР с коаксиальным и планарным расположением электродов. В качестве плазмообразующего газа использовался кислород. Концентрацию нефтепродуктов (НП) и альдегидов определяли флуориметрическим методом. Контроль одноосновных карбоновых кислот проводился фотометрическим методом с использованием спектрофотометра. Определение содержания $\mathrm{CO}$ и $\mathrm{CO}_{2}$ в газовой фазе на выходе из реактора, проводилось методом газовой хроматографии с помощью пламенно-ионизационного детектора. Концентрация озона, образующегося в разрядной зоне реактора, определялась методом абсорбционной спектроскопии по поглощению света на $\lambda=254$ нм.

Для оценки количества циклов «сорбция-десорбция» исследуемого адсорбента были проведены эксперименты при многократных обработках в реакторах ДБР с различным расположением электродов. Установлено, что при многократной регенерации загрязненного адсорбента его сорбционные свойства снижаются. Однако даже при максимальном числе циклов регенерации, при использовании каждого из реакторов, сорбционная емкость лишь после 5 циклов достигает величины сорбционной емкости, характерной для исходного диатомита (2.63 мг/г). Таким образом, оптимальное число циклов сорбции/десорбции составляет 5, и ДБР можно использовать для многократной регенерации одного и того же сорбента.

Коаксиальная система является более эффективной, чем планарная, как по эффективности деструкции, так и по величине сорбционной емкости сорбента после первоначальной обработки диатомита ДБР (в 1.4 раза), что, вероятно, связано с тем, что в коаксиальной системе распределение сорбента в зоне горения разряда позволяет более эффективно взаимодействовать активным частицам плазмы с сорбентом. Сравнение результатов обработки сорбента в различных реакторах ДБР показало, что коаксиальная система является более энергоэффективной - удельные затраты энергии на регенерацию сорбента в 3 раза ниже, чем в планарной системе. Единственным достоинством планарной системы является меньшее время обработки адсорбента.

Существенным достоинством обработки загрязнённых НП сорбентов является не только возможность их регенерации, но и высокая степень минерализации исходных токсикантов (96-98 \%). Установлено, что в невысоких временах обработки (до 300 с) продукты деструкции НП (такие как карбоновые кислоты и альдегиды) не полностью разлагаются и постепенно накапливаются в объеме сорбента, что приводит к снижению сорбционной емкости при повторном использовании. Однако увеличение времени воздействия разряда на загрязнённый сорбент приводило к тому, что сорбированные на нем альдегиды и кислоты, окисляются до диоксида углерода. Для полной регенерации сорбента необходимо 40 минут обработки в разряде, а вклад $\mathrm{CO}_{2}$ в баланс по «углероду» составляет 94 \% от углерода, 
содержащего в НП до обработки в ДБР. Эксперименты показали, что концентрации озона в системе недостаточно для полного окисления НП. Таким образом, в процессе окисления должны участвовать другие активные частицы, образующиеся в зоне разряда, например, $\mathrm{O}, \mathrm{OH}^{*}$ и Н .

Ключевые слова: диатомит, плазма, регенерация, нефтепродукты, ресурсосбережение, водоочистка.

\section{Введение}

С развитием научно-технического прогресса постоянно увеличивается нагрузка на окружающую среду, в частности, на гидросферу, загрязнение которой происходит за счет попадания в водные объекты различных веществ, как органической, так и неорганической природы. Удаление загрязняющих веществ до значений, соответствующих нормативным требованиям, является сложной и дорогостоящей задачей. К одному из наиболее известных и распространённых методов очистки сточных вод можно отнести адсорбционный. Однако ему свойственен ряд недостатков, таких как необходимость в постоянном контроле сорбционной емкости сорбента, дополнительные затраты на регенерацию, а также утилизация уловленных веществ и отработанного сорбента.

Перспективным направлением развития сорбционной техники является поиск новых эффективных способов осуществления сорбционно-десорбционных процессов, связанных с использованием нетрадиционных для данной области видов энергии и путей ее передачи сорбционным материалам. В последнее время внимание многих исследователей привлекают методы химии высоких энергий, в частности диэлектрический барьерный разряд (ДБР) [1]. Плазменно-адсорбционная очистка воды с последующей (или одновременной) регенерацией сорбентов с помощью ДБР является новой и малоизученной областью, поэтому исследование процессов обработки сорбентов в барьерном разряде является актуальной и важной задачей.

Целью работы являлось выявление закономерностей процессов регенерации диатомита в плазме диэлектрического барьерного разряда, и особенностей воздействия плазмы на поверхностные свойства сорбента (диатомита марки СМД-Сорб) в реакторах ДБР планарного и коаксиального типов.

\section{Теоретическая часть}

Под ДБР в настоящее время понимают разряд, возникающий в газе под действием приложенного к электродам переменного напряжения, при этом хотя бы один из электродов должен быть покрыт диэлектриком [2]. Использование плазмы ДБР также включает стерилизацию бактерий в почве [3], активацию полимеров [4], очистку и модификацию поверхности материалов [5-6]. Также одним из наиболее важных аспектов применения диэлектрического барьерного разряда, является защита окружающей среды от загрязнения, что в первую очередь связано с тем, что в ДБР происходит генерация большого количества различных активных частиц (ионы, радикалы, О 3 ), которые peaгируют с большинством органических соединений $[7,8]$. Регенерация в плазме ДБР загрязненных сорбентов, ввиду эффективного взаимодействия активных частиц с сорбированными органическими веществами, позволит их многократно повторно использовать [9-11].

Регенерация адсорбентов с помощью барьерного разряда является новой и малоизученной областью, поэтому изучение процессов, происходящих при обработке загрязнённых сорбентов в плазме является весьма актуальной и важной задачей. Стоит отметить, что исследования в данной области уже проводятся. В работе [12] приведены результаты экспериментов по регенерации активированного угля, загрязнённого пентахлорфенолом в плазме двойного диэлектрического барьерного разряда. Эффективность разложения пентахлорфенола с поверхности гранулированного активированного угля составила $65 \%$, а восстановление сорбционной емкости адсорбента - 87\% [12]. 
В работе [13] представлена экспериментальная установка ДБР для регенерации активированного угля, загрязненного фенолом. Установка позволяет обрабатывать до 1200 грамм загрязненного сорбента, с эффективностью удаления фенола с поверхности сорбента до 70\%, и восстановлению сорбционной емкости сорбента до 94\%. В ходе экспериментов было установлено, что максимальное количество циклов «адсорбция/регенерация» составило четыре. Однако, ни продукты деструкции и, соответственно, вероятный механизм разложения фенола, авторами установлен не был [13]. В работе [14] ДБР был применен для обработки сепиолита, загрязненного красителем малахитовым зеленым. Было изучено влияние на адсорбционную емкость сорбента таких параметров ДБР, как время воздействия плазмы, тип плазмообразующего газа, $\mathrm{pH}$ раствора, содержащего краситель. Было установлено, что при обработке сорбента в ДБР происходит химическая и топографическая модификация его поверхности, а величина его удельной поверхности после обработки в ДБР увеличилась с 267 до $309 \mathrm{~m}^{2} / \Gamma$ [14].

Таким образом, обработка загрязненных сорбентов в ДБР позволяет регенерировать и улучшать их адсорбционные свойства, однако кинетические закономерности и механизм процесса деструкции загрязнителя с поверхности сорбента изучены недостаточно.

\section{Экспериментальная часть}

Плазмохимический реактор планарного типа представлял собой стеклянный сосуд цилиндрической формы, внутренний диаметр которого составлял 60 мм. Толщина диэлектрического барьера между электродом и обрабатываемым сорбентом составляла 3 мм. Сосуд герметично закрывался крышкой, в которой был вмонтирован электрод, изготовленный из алюминия ( $\varnothing 30$ мм). В качестве плазмообразующего газа использовался технический кислород. Барьерный разряд возбуждался от высоковольтного трансформатора, величина приложенного к электродам напряжения составляла 10.628.4 кВ.

Реактор ДБР, реализующий коаксиальное расположение электродов, представлял собой стеклянную трубку, выступающей в качестве диэлектрика, внутри которой размещался внутренний электрод. Внешний электрод, представлял собой фольгу, закреплённую на внешней стенке реактора. Электроды были выполнены из алюминия. Сорбент внутри ячейки удерживался в разрядной зоне с помощью перфорированных фторопластовых колец. Барьерный разряд возбуждался от высоковольтного трансформатора, аналогичного для установки планарного типа. Более подробное описание реакторов ДБР, а также параметры проведения эксперимента представлены в $[15,16]$.

В качестве нефтепродуктов (НП) использовалось моторное масло М-8В. Концентрация нефтепродуктов определялась флуориметрическим методом с использованием флуориметра «Флюорат-02» (Люмекс, Россия) [17]. Для определения приближенной молекулярной массы НП использовалась формула Крейга [18]:

$$
M=44.29 \times \rho^{298} /\left(1.03-\rho^{298}\right),
$$

где $\rho^{298}$ - плотность масла (905 кг м $\left.{ }^{3}\right)$ при 298 К, М - молярная масса (г/моль). Расчет по этому соотношению дает приближенную формулу масла М8-B - $\mathrm{C}_{22} \mathrm{H}_{38}$ и М=302 г/моль.

Концентрацию альдегидов определяли флуориметрическим методом (флуориметр «Флюорат 2-М» (ООО «Люмэкс», Россия)) [19]. Контроль одноосновных карбоновых кислот проводился фотометрическим методом с использованием спектрофотометра («UNICO, мод. 2804») [20]. Определение содержания $\mathrm{CO}$ и $\mathrm{CO}_{2}$ в газовой фазе на выходе из реактора, проводилось методом газовой хроматографии с помощью пламенно-ионизационного детектора.

Концентрация озона, образующегося в разрядной зоне реактора, определялась методом абсорбционной спектроскопии 




Рис. 1. Изменение сорбционной емкости в зависимости от количества циклов регенерации: 1 - коаксиальная система,

2 - планарная система, 3 - исходная емкость. Fig. 1. Change in sorption capacity depending on the number of regeneration cycles: 1 - coaxial system, 2 - planar system, 3 - initial capacity.

по поглощению света на $\lambda=254$ нм [21].

Определение сорбционной емкости исследуемого образца сорбента проводилось по формуле:

$$
J=\frac{V_{0} \times\left(C-C_{0}\right)}{m}, \mathrm{M \Gamma} / \Gamma
$$

где J - сорбционная емкость сорбента, мг/г, $\mathrm{V}_{0}$ - объём пробы модельного раствора, пропущенного через сорбент, дм ${ }^{3}$; $\mathrm{C}_{0}$ и $\mathrm{C}$ - начальная и конечная концентрации вещества соответственно, мг/дм³ $\mathrm{m}$ - масса образца сорбента, г.

Условия проведения эксперимента: начальная концентрация нефтепродук-

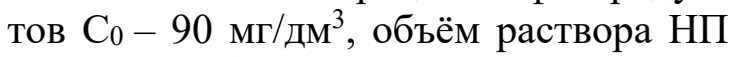
$\left(\mathrm{V}_{0}\right)-100 \mathrm{~cm}^{3}$, масса сорбента $(\mathrm{m})-2$ г.

Для оценки удельных энергозатрат, затрачиваемых на процесс обработки сорбентов (G, кДж/кг), использовалась формула:

$$
\mathrm{G}=(\mathrm{U} \cdot \mathrm{I} \cdot \mathrm{t}) / \mathrm{m}
$$

где $\mathrm{U}$ - напряжение, подводимое к реактору ДБР, кВ; I - ток разряда, мА; $\mathrm{m}$ масса регенерируемого сорбента, г; $\mathrm{t}$ - время обработки сорбента в ДБР, с.

\section{Обсуждение результатов}

Для оценки количества циклов «сорбция-десорбция» исследуемого адсорбента были проведены эксперименты при

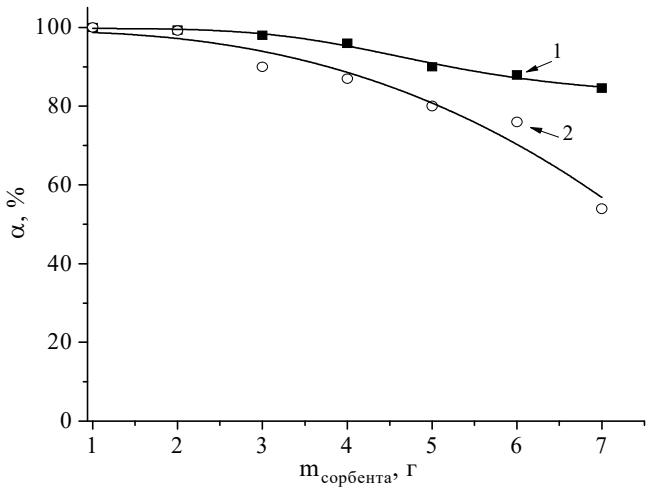

Рис. 2. Зависимость эффективности деструкции НП с поверхности сорбента от массы обрабатываемого диатомита: 1 - коаксиальная система, 2 - планарная система.

Fig. 2. Dependence of the efficiency of destruction of OP from the surface of the sorbent on the mass of the processed diatomite:

1 - coaxial system, 2 - planar system.

многократной обработке в реакторах ДБР с различным расположением электродов (рис. 1). Загрязненный образец обрабатывали плазмой в реакторах ДБР, а затем промывали гексаном для удаления нефтепродуктов (коэффициент экстракции составлял порядка 0.95). Затем образец высушивали при температуре $105^{\circ} \mathrm{C}$ до достижения постоянной массы (около 12 часов) и повторно насыщали раствором нефтепродуктов для определения сорбционной емкости. Образец снова подвергали воздействию разряда при оптимальных параметрах обработки для каждого из реакторов.

Установлено, что при многократной регенерации загрязненного адсорбента его сорбционные свойства снижаются. Однако даже при максимальном числе циклов регенерации, при использовании каждого из реакторов, сорбционная емкость лишь после 5 циклов достигает величины сорбционной емкости, характерной для исходного диатомита (2.63 мг/г). Таким образом, оптимальное число циклов сорбции/десорбции составляет 5, и ДБР можно использовать для многократной регенерации одного и того же сорбента. Скорее всего, такая ситуация обусловлена тем, что продукты распада не 
Таблица 1. Сравнение оптимальных параметров обработки загрязненного адсорбента в реакторах ДБР с коаксиальным и планарным расположением электродов

Table 1. Comparison of the optimal processing parameters of contaminated adsorbent in DBR reactors with a coaxial and planar arrangement of electrodes

\begin{tabular}{|c|c|c|c|c|c|c|c|}
\hline $\begin{array}{c}\text { Тип реактора } \\
\text { ДБР }\end{array}$ & $\mathrm{J}, \mathrm{мг/ \Gamma}$ & $\begin{array}{c}\mathrm{P}, \\
\mathrm{BT} / \mathrm{cm}^{3}\end{array}$ & $\begin{array}{c}\text { Расход } \\
\text { газа, } \\
\text { дм } 3 \text { мин }\end{array}$ & $\begin{array}{c}\mathrm{t}, \\
\text { мин }\end{array}$ & $\begin{array}{c}\text { N циклов сорб- } \\
\text { ция/десорбция }\end{array}$ & $\mathrm{M}, \Gamma$ & $\begin{array}{c}\mathrm{G}, \text { кДж/г } \\
\text { сор- } \\
\text { бента }\end{array}$ \\
\hline $\begin{array}{c}\text { Коаксиаль- } \\
\text { ный }\end{array}$ & 4.8 & 1.2 & 0.5 & 5 & 5 & 6 & 0.016 \\
\hline Планарный & 3.4 & 8.9 & 0.5 & 1 & 5 & 4 & 0.050 \\
\hline
\end{tabular}

полностью удаляются в течение времени обработки и постепенно накапливаются на поверхности, снижая ее сорбционную емкость.

Результаты экспериментов, приведенные на рис. 2 показывают, что коаксиальная система является более эффективной, чем планарная, как по эффективности деструкции (в зависимости от массы сорбента в реакторе от 5 до 40\%), так и по величине сорбционной емкости сорбента после первоначальной обработки диатомита ДБР (в 1.4 раза), что, вероятно, связано с тем, что в коаксиальной системе распределение сорбента в зоне горения разряда позволяет более эффективно взаимодействовать активным частицам плазмы с сорбентом (включая объёмные процессы).

Сравнение результатов обработки сор-



Рис. 3. Зависимость концентрации карбоновых кислот (1) и альдегидов (2) от времени обработки в плазменно-сорбционном процессе.

Fig. 3. Dependence of the concentration of carboxylic acids (1) and aldehydes (2) on the treatment time during the plasma sorption process. бента в различных реакторах ДБР представлено в табл. 1. Оценки показали, что коаксиальная система помимо указанных выше преимуществ, является более энергоэффективной - удельные затраты энергии на регенерацию сорбента в 3 раза ниже, чем в планарной системе. Единственным достоинством планарной системы является меньшее время обработки адсорбента.

Существенным достоинством обработки загрязнённых НП сорбентов является не только возможность их регенерации, но и высокая степень минерализации исходных токсикантов, которая может достигать 96-98\%. Было установлено, что в невысоких временах обработки (до 300c) продукты деструкции НП (такие как карбоновые кислоты (КК) и альдегиды (рис. 3)) не полностью разлагаются и постепенно накапливаются в объеме



Рис. 4. Изменение концентрации $\mathrm{CO}_{2}(1)$ и эффективности разложения НП (2)

Fig. 4. Change in $\mathrm{CO}_{2}$ concentration (1) and the efficiency of OP decomposition (2) 
сорбента, что приводит к снижению сорбционной емкости при повторном использовании. Однако увеличение времени воздействия разряда на загрязнённый сорбент приводило к тому, что сорбированные на нем альдегиды и КК, окисляются до диоксида углерода. Изменение концентрации $\mathrm{CO}_{2}$ в системе при обработке сорбента и эффективность деструкции НП от времени приведено на рис. 4. Из представленных данных видно, что для полной регенерации сорбента необходимо 40 минут обработки в разряде, а вклад $\mathrm{CO}_{2}$ в баланс по «углероду» составляет $94 \%$ от углерода, содержащего в НП до обработки в ДБР.

Одним из основных окислительных агентов, образующихся в ДБР, является озон. Для полного окисления 1 моль НП в соответствии с реакцией
17 мкмоль, то есть для полного окисления требуется 357 мкмоль О3, следовательно, концентрации озона в системе недостаточно для их окисления. Т.е. в процессе окисления должны участвовать другие активные частицы, образующиеся в зоне разряда, например, такие как $\mathrm{O}, \mathrm{OH}^{*}$ и $\mathrm{H}^{*}$. Полученные данные позволяют предположить возможный механизм деструкции НП, который может быть представлен схемой 1.

\section{Заключение}

Таким образом, можно сделать вывод, что регенерация сорбентов с использованием реакторов ДБР как с планарным, так и коаксиальным расположением электродов является эффективной. Основными продуктами деструкции НП являются карбоновые кислоты, альдегиды и диок-

Схема 1/Cheme 1

1 этап: образование алкильных радикалов в реакииях с активными частицами плазмы $\sim \mathrm{CH}_{2}-\mathrm{CH}_{2}-\mathrm{CH}_{2} \sim+\mathrm{OH} \rightarrow \sim \mathrm{CH}_{2}-\mathrm{CH}-\mathrm{CH}_{2} \sim+\mathrm{H}_{2} \mathrm{O}$, $\sim \mathrm{CH}_{2}-\mathrm{CH}_{2}-\mathrm{CH}_{2} \sim+\mathrm{O} \rightarrow \sim \mathrm{CH}_{2}-\mathrm{CH}-\mathrm{CH}_{2} \sim+\cdot \mathrm{OH}$, $\sim \mathrm{CH}_{2}-\mathrm{CH}_{2}-\mathrm{CH}_{2} \sim+\mathrm{H} \rightarrow \sim \mathrm{CH}_{2}-\mathrm{CH}-\mathrm{CH}_{2} \sim+\mathrm{H}_{2}$.

2 этап: окисления алкильных радикалов (например, с О( $\left.{ }^{3} P\right)$ ) с образованием алкоксирадикалов и последующим их разрушением до КК

$$
\begin{aligned}
\sim \mathrm{CH}_{2}-\mathrm{CH}^{-}-\mathrm{CH}_{2} \sim & +\mathrm{O} \rightarrow \sim \mathrm{CH}_{2}-\mathrm{CHO}^{-}-\mathrm{CH}_{2} \sim \rightarrow \sim \mathrm{CH}_{2}-\mathrm{C}=\mathrm{O}+\mathrm{CH}_{3} \sim, \\
\sim \mathrm{CH}_{2}-\mathrm{C} & =\mathrm{O}+\mathrm{OH} \rightarrow \sim \mathrm{CH}_{2}-\mathrm{COOH} .
\end{aligned}
$$

3 этап: превращение КК до альдегидов и окисление последних до $\mathrm{CO}_{2}$

$$
\begin{gathered}
\sim \mathrm{CH}_{2}-\mathrm{COOH}+\mathrm{O} \rightarrow \sim \mathrm{CH}_{2}-\mathrm{CH}-(\mathrm{C}=\mathrm{O})-\mathrm{OH}+\mathrm{OH} ; \\
\sim \mathrm{CH}_{2}-\mathrm{COOH}+\mathrm{OH} \rightarrow \sim \mathrm{CH}_{2}-\mathrm{CH}^{-}-(\mathrm{C}=\mathrm{O})-\mathrm{OH}+\mathrm{H}_{2} \mathrm{O} ;
\end{gathered}
$$$$
\mathrm{CH}_{2}-\mathrm{CH}^{-}-(\mathrm{C}=\mathrm{O})-\mathrm{OH}+\mathrm{O} \rightarrow \sim \mathrm{CH}_{2}-\mathrm{CHO}^{-}-(\mathrm{C}=\mathrm{O})-\mathrm{OH} \text {; }
$$$$
\sim \mathrm{CH}_{2}-\mathrm{CH}-(\mathrm{C}=\mathrm{O})-\mathrm{OH}+\mathrm{O}_{3} \rightarrow \sim \mathrm{CH}_{2}-\mathrm{CHO}-(\mathrm{C}=\mathrm{O})-\mathrm{OH}+\mathrm{O}_{2} \text {, }
$$$$
\mathrm{CH}_{2}-\mathrm{CHO}_{-}-(\mathrm{C}=\mathrm{O})-\mathrm{OH} \rightarrow(\mathrm{C}=\mathrm{O})-\mathrm{OH}+\sim \mathrm{CH}_{2}-\mathrm{CHO} \text {, }
$$$$
(\mathrm{C}=\mathrm{O})-\mathrm{OH}+\mathrm{OH} \rightarrow \mathrm{H}_{2} \mathrm{CO}_{3} \rightarrow \mathrm{H}_{2} \mathrm{O}+\mathrm{CO}_{2} ; \sim \mathrm{CH}_{2}-\mathrm{CHO}+\mathrm{OH} \rightarrow \sim \mathrm{CH}_{2}-(\mathrm{C}=\mathrm{O})+\mathrm{H}_{2} \mathrm{O} \text {; }
$$$$
\sim \mathrm{CH}_{2}-(\mathrm{C}=\mathrm{O}) \rightarrow \mathrm{CO}+\sim \mathrm{CH}_{2} ; \sim \mathrm{CH}_{2}-(\mathrm{C}=\mathrm{O})+\mathrm{O}_{3} \rightarrow \mathrm{CO}_{2}+\sim \mathrm{CH}_{2}{ }^{-}+\mathrm{O}_{2} ;
$$$$
\sim \mathrm{CH}_{2}+\mathrm{O}_{2} \rightarrow \mathrm{CH}_{2} \mathrm{O} ; \mathrm{CH}_{2} \mathrm{O}+\mathrm{O} \rightarrow \mathrm{CHO}+\mathrm{OH} \text {; }
$$

$\mathrm{CHO}+\mathrm{O}{ }_{2} \rightarrow \mathrm{CO} 2(\mathrm{CO})+\mathrm{OH}\left(\mathrm{H}_{2} \mathrm{O}\right): \mathrm{CO}+\mathrm{O} \rightarrow \mathrm{CO}_{2}$

$\mathrm{C}_{22} \mathrm{H}_{38}+21 \mathrm{O}_{3} \rightarrow 22 \mathrm{CO}_{2}+19 \mathrm{H}_{2} \mathrm{O}$

требуется 21 моль Оз. Экспериментально было определено, что максимальное снижение концентрации озона для окисления НП (при концентрации равной 333 мкмоль/дм $\left.{ }^{3}\right)$ в ДБР составляет $7.5 \cdot 10^{16} \mathrm{~cm}^{-3}$ или 120 мкмоль при расходе $\mathrm{O}_{2} 0.2$ дм $^{3} /$ мин (время обработки 5 мин). Содержание НП в системе составляете сид углерода. Обработка загрязнённого сорбента в течении 40 мин позволяет достигать степени минерализации не менее 96\%. Результаты экспериментов показали, что коаксиальный тип реактора является более эффективным для регенерации загрязненных сорбентов, чем планарный, как по эффективности деструкции НП, так и по энергетическим затратам.

Работа выполнялась при поддержке гранта РФФИ (грант № 18-08-01239) и государственного задания на выполнение НИР: тема № FZZW-2020-0010. 


\section{Список литературы}

1. Fang Z., Qiu Y.C., Sun Y.Z., Wang H. et al. // J. Electrostat. 2008. Vol. 66, No. 7-8. pp. 421-426.

2. Самойлович В.Т., Гибалов В.И., Козлов К.В. Физическая химия барьерного разряда. М. МГУ. 1989. 176 с.

3. Takayama M., Ebihara K., Stryczewska H., Ikegami T. et al. // Thin Solid Films. 2006. Vol. 506-507. pp. 396-399.

4. Klages C.P., Hinze A., Lachmann K., Berger C. et al. // Plasma Process. Polym., 2007. Vol. 4. pp. 208-218.

5. Xu X. // Thin Solid Films. 2001. Vol. 390. pp. 237-242.

6. Kodama S., Habaki H., Sekiguchi H., Kawasaki J. // Thin Solid Films. 2002. Vol. 407. pp. 151-155.

7. Francke K.P., Miessner H., Rudolph R. // Catal. Today. 2000. Vol. 59. pp. 411-416.

8. Magureanu M., Mandache N.B., Eloy P., Gaigneaux E.M. et al., Appl. Catal. 2005. Vol. 61. pp. 12-20.

9. Yamagata Y., Niho K., Inoue K. et al. // Jpn. J. Appl. Phys. 2006. Vol. 45. pp. 82518254.

10. Yang J.T., Shi Y., Li W., Wang X. et al. // Abstr. Pap. Am. Chem. Soc. 2006. Vol. 231. p. 148.

11. Zhang Y.Z., Zheng J.T., Qu X.F., Chen H.G. // J. Colloid Interface Sci. 2007. Vol. 316. pp. 523-530.

12. Lu N., Li J., Wang X., Wang T. et al. Plasma Chemistry and Plasma Processing,
2012, Vol. 3. No 1. pp. 109-121.

13. Tang S. et al.// Separation and purification technology. 2012. Vol. 95. pp. 73-79.

14. Kaya M., Dilekoğlu M.F., Şahin Ö., Saka C. // Plasma Chemistry and Plasma Processing. 2016. Vol. 36. No 6. pp. 1417-1430.

15. Grinevich V.I., Rybkin V.V., Lyubimov V.A., Gushchin A.A. // Izvestiya Vysshikh Uchebnykh Zavedenii. Seriya Khimiya $i$ Khimicheskaya Tekhnologiya. 2017. Vol. 60. No 8. pp. 20-27.

16. Gushchin A.A., Grinevich V.I., Gusev G.I., Kvitkova E.Yu. // Plasma Chemistry and Plasma Processing. 2017. Vol. 38. No 5. pp. 1021-1033.

17. ПНД Ф 14.1:2:4.129-98. Методические указания по измерению массовой концентрации нефтепродуктов в пробах питьевой воды и воды поверхностных и подземных источников водопользования.

18. Казакова Л.П., Крейн С.Э. Физико-химические основы производства нефтяных масел. М. Химия. 1978. Т. 2. 320 с.

19. ПНД Ф 14.1:2:4.187-02 Методика измерения массовой концентрации формальдегида в пробах природных, питьевых и сточных вод флуориметрическим методом на анализаторе жидкости Флюорат- 02 .

20. Симонов В.А., Нехорошева Е.В., Заворовская Н.А. Анализ воздушной среды при переработке полимерных материалов. Л. Изд. Химия.1988. С. 19.

21. Parkinson W.H., Yoshino K., Freeman D.E. // Smithsonian Institution Astrophysical Observatoryю МА. 1988. p. 2138.

\title{
Effects of dielectric barrier discharge on diatomite contaminated with oil products
}

\author{
(C) 2021 Gusev G.I., Gushchin A.A., Grinevich V.I., \\ Izvekova T.V., Sharonov A.V. \\ Ivanovo State University of Chemistry and Technology, Ivanovo, Russian Federation
}

\begin{abstract}
Recently, the attention of many researchers has been attracted by the methods of high-energy chemistry, in particular, the dielectric barrier discharge (DBD). The plasma-adsorption of water treatment with subsequent (or simultaneous) regeneration of sorbents using DBR is a new and poorly studied area, therefore, the study of the processes of sorbent treatment in a barrier discharge is an urgent and important task.

The aim of this study was the identification of the regularities of the processes of regeneration of diatomite in the dielectric barrier discharge plasma, and the features of the effect of plasma on the surface properties of the sorbent (diatomite of SMD-Sorb brand) in planar and coaxial DBR reactors.

We used two DBR reactors with coaxial and planar electrodes. Oxygen was used as a plasma-forming gas. The concentration of oil products (OP) and aldehydes was determined by the fluorimetric method. The
\end{abstract}


control of monobasic carboxylic acids was carried out photometrically using a spectrophotometer. The determination of $\mathrm{CO}$ and $\mathrm{CO}_{2}$ contents in the gas phase at the outlet of the reactor, was carried out by gas chromatography using a flame ionization detector. The concentration of ozone formed in the discharge zone of the reactor was determined by the absorption spectroscopy based on light absorption at $\lambda=254 \mathrm{~nm}$.

For the estimation of the number of "sorption-desorption" cycles of the studied adsorbent, experiments were carried out with multiple treatments in DBR reactors with different arrangements of electrodes. It was found that with repeated regeneration of the contaminated adsorbent, its sorption properties decreased. However, even with the maximum number of regeneration cycles, when using each of the reactors, the sorption capacity only after 5 cycles reached the value of the sorption capacity of the original diatomite $(2.63 \mathrm{mg} / \mathrm{g})$. Thus, the optimal number of sorption /desorption cycles was 5, and DBR can be used for multiple regenerations of the same sorbent.

The coaxial system is more efficient than the planar one, both in terms of the destruction efficiency and the sorption capacity of the sorbent after the initial treatment of diatomite by DBR (1.4 times), which is probably due to the fact that in the coaxial system the sorbent distribution in the discharge combustion zone allows a more effective interaction of active plasma particles with the sorbent. The comparison of the results of sorbent processing in various DBR reactors showed that the coaxial system is more energy efficient, the specific energy consumption for sorbent regeneration was 3 times lower than in the planar system. The only advantage of the planar system was the shorter processing time of the adsorbent.

An essential advantage of the processing of sorbents contaminated with OP is not only the possibility of their regeneration, but also a high degree of mineralization of the original toxicants (96-98\%). It was found that, during short processing times (up to $300 \mathrm{~s}$ ), the products of OP destruction (such as carboxylic acids and aldehydes) did not completely decompose and gradually accumulated in the volume of the sorbent, causing a decrease in the sorption capacity upon repeated use. However, an increase in the exposure time of the discharge on the contaminated sorbent led to the oxidation of sorbed aldehydes and acids into carbon dioxide. Full regeneration of the sorbent required $\sim 40$ minutes of treatment in the discharge, and the contribution of $\mathrm{CO}_{2}$ in the "carbon" balance was $94 \%$ of the carbon contained in the OP before processing in the DBR. Experiments have shown that ozone concentration in the system was insufficient for the complete oxidation of the OP. Thus, other active particles formed in the discharge zone, for example, $\mathrm{O}, \mathrm{OH}$, and $\mathrm{H}^{-}$should be involved in the oxidation process.

Keywords: diatomite, plasma, regeneration, oil products, efficient use of resources, water treatment.

\section{References}

1. Fang Z., Qiu Y.C., Sun Y.Z., Wang H. et al., J. Electrostat, 2008, Vol. 66, No 7-8, pp. 421-426.

2. Samoilovich V.T., Gibalov V.I., Kozlov K.V., Fizicheskaya khimiya bar'ernogo razryada, $M ., M S U, 1989,176 \mathrm{p}$.

3. Takayama M., Ebihara K., Stryczewska H., Ikegami T. et al., Thin Solid Films, 2006, Vol. 506-507, pp. 396-99.

4. Klages C.P., Hinze A., Lachmann K., Berger C. et al., Plasma Process. Polym., 2007, Vol. 4, pp. 208-218.

5. Xu X., Thin Solid Films, 2001, Vol. 390, pp. 23-242.

6. Kodama S., Habaki H., Sekiguchi H., Kawasaki J., Thin Solid Films, 2002, Vol. 407, pp. 151-155.

7. Francke K.P., Miessner H., Rudolph R., Catal. Today, 2000, Vol. 59, pp. 411-416.

8. Magureanu M., Mandache N.B., Eloy P., Gaigneaux E.M. et al., Appl. Catal., 2005, Vol. 61, pp. 12-20.

9. Yamagata Y., Niho K., Inoue K. et al., Jpn. J. Appl. Phys., 2006, Vol. 45, pp. 8251-8254.

10. Yang J.T., Shi Y., Li W., Wang X. et al.,
Abstr. Pap. Am. Chem. Soc., 2006 Vol. 231, p. 148.

11. Zhang Y.Z., Zheng J.T., Qu X.F., Chen H.G., J. Colloid Interface Sci., 2007, Vol. 316, pp. 523-530.

12. Lu N., Li J., Wang X., Wang T. et al., Plasma Chemistry and Plasma Processing, 2012, Vol. 32, No 1, pp. 109-121.

13. Tang S. et al., Separation and purification technology, 2012, Vol. 95, pp. 73-79.

14. Kaya M., Dilekoğlu M. F., Şahin Ö. \& Saka C., Plasma Chemistry and Plasma Processing, 2016, Vol. 36, No 6, pp. 1417-1430.

15. Grinevich V.I., Rybkin V.V., Lyubimov V.A., Gushchin A.A., Izvestiya Vysshikh Uchebnykh Zavedenii. Seriya Khimiya $i$ Khimicheskaya Tekhnologiya, 2017, Vol. 60, No 8, pp. 20-27.

16. Gushchin A.A., Grinevich V.I., Gusev G.I., Kvitkova E.Yu., Rybkin V.V., Plasma Chemistry and Plasma Processing, 2017, Vol. 38, No 5, pp. 1021-1033.

17. PND F 14.1:2:4.129-98. Metodiche-skie ukazaniya po izmereniyu massovoy kontsentratsii nefteproduktov $\mathrm{v}$ probakh pit'evoy vody 
i vody poverkhnostnykh i podzemnykh istochnikov vodopol'zovaniya.

18. Kazakova L.P., Kreyn S.E. Fizikokhimicheskie osnovy proizvodstva neftya-nykh masel. M. Khimiya. 1978. Vol. 2. 320 p.

19. PND F 14.1:2:4.187-02 Metodika izmereniya massovoy kontsentratsii for-mal'degida $\mathrm{v}$ probakh prirodnykh, pit'e-vykh i stochnykh vod fluorimetricheskim metodom na analizatore zhidkosti Flyuo-rat-02.

Гусев Григорий Игоревич - младший научный сотрудник, ФГБОУ ВО «Ивановский государственный химико-технологический университет», Иваново

Гущин Андрей Андреевич - к.х.н., доцент, заведующий кафедрой промышленной экологии, ФГБОУ ВО «Ивановский государственный химико-технологический университет», Иваново

Гриневич Владимир Иванович - д.Х.н., професcop, кафедра промышленной экологии ФГБОУ ВО «Ивановский государственный химико-технологический университет», Иваново

Извекова Татьяна Валерьевна - к.Х.Н., доцент, кафедра промышленной экологии ФГБОУ ВО «Ивановский государственный химико-технологический университет», Иваново

Шаронов Александр Винидиктович - студент кафедры промышленной экологии ФГБОУ ВО «Ивановский государственный химико-технологический университет», Иваново
20. Simonov V.A., Nekhorosheva E.V., Zavorovskaya N.A. Analiz vozdushnoy sredy pri pererabotke polimernykh materialov. L. Izd. KHimiya.1988. 19 p.

21. Parkinson W.H., Yoshino K., Freeman D.E., Smithsonian Institution Astrophysical Observatory, MA, 1988, 2138 p.

Gusev Grigory I. - research fellow, Ivanovo State University of Chemical Technology, Ivanovo, email: grisha.gusev.05@mail.ru

Gushchin Andrey A. - Ph.D. (chemistry), associate prof., Head of the department of industrial Ecology, Ivanovo State University of Chemical Technology, Ivanovo, e-mail: a_guschin@bk.ru

Grinevich Vladimir I. - doctor of chemistry, professor, department of industrial ecology, Ivanovo State University of Chemical Technology, Ivanovo

Izvekova Tatiana V. - Ph.D. (chemistry), associate prof., department of Industrial Ecology, Ivanovo State University of Chemical Technology, Ivanovo

Sharonov Alexander V. - student of the department of industrial ecology, Ivanovo State University of Chemical Technology, Ivanovo 